\title{
Gut Microbiota: A Potential Target for Cancer Interventions
}

\author{
Hu Zhou ${ }^{1,2, *}$ \\ Yuan Yuan ${ }^{1-3, *}$ \\ Haorun Wang ${ }^{1,2}$ \\ Wei Xiang ${ }^{1,2}$ \\ shenjie $\mathrm{Li}^{1,2}$ \\ Haowen Zheng ${ }^{1,2}$ \\ Yuqi Wen ${ }^{1,2}$ \\ Yang Ming ${ }^{1,2}$ \\ Ligang Chen ${ }^{1,2,4,5}$ \\ Jie Zhou (iD) ${ }^{1,2,4,5}$
}

'Department of Neurosurgery, Affiliated Hospital of Southwest Medical University, Luzhou, Sichuan, People's Republic of China; ${ }^{2}$ Sichuan Clinical Research Center for Neurosurgery, Luzhou, Sichuan, People's Republic of China; ${ }^{3}$ Department of Operation and Anaesthesia, Yibin First People's Hospital, Yibin, Sichuan, People's Republic of China; ${ }^{4}$ Academician (Expert) Workstation of Sichuan Province, Luzhou, Sichuan, People's Republic of China; ${ }^{5}$ Neurological Diseases and Brain Function Laboratory, Luzhou, Sichuan, People's Republic of China

*These authors contributed equally to this work
Correspondence: Jie Zhou Email zj000718@yeah.net

\begin{abstract}
The gut microbiota plays a crucial role in many physiological processes in the human body. Dysbiosis can disrupt the intestinal barrier and alter metabolism and immune responses, leading to the development of diseases. Over the past few decades, evidence has accumulated linking changes in the composition of the gut microbiota to dozens of seemingly unrelated conditions, including cancer. Overall, the gut microbiota mainly affects the occurrence and development of cancer by damaging host DNA, forming and maintaining a pro-inflammatory environment, and affecting host immune responses. In addition, the gut microbiota can also affect the efficacy and toxicity of chemotherapy, radiotherapy, and immunotherapy. Scientists attempt to improve the efficacy and decrease the toxicity of these treatment modalities by fine-tuning the gut microbiota. The aim of this review is to assist researchers and clinicians in developing new strategies for the detection and treatment of tumors by providing the latest information on the intestinal microbiome and cancer, as well as exploring potential application prospects and mechanisms of action.
\end{abstract}

Keywords: intestinal flora, carcinogenesis, mechanism, therapy, fecal bacteria transplantation

\section{Introduction}

Currently, cancer is the leading cause of death worldwide, and its morbidity and mortality rates continue to rise in parallel with the increasing and aging population. ${ }^{1}$ It is estimated that $\leq 20 \%$ of the global cancer burden is caused by microorganisms, such as Helicobacter pylori, Clostridium difficile, Epstein-Barr virus, human papillomavirus, and other pathogens associated with cancer. ${ }^{2}$ As the largest microbial reservoir in the human body, a balanced gut microbiota is positively associated with health. However, dysbiosis (ie, alterations in microbial diversity and/or function) can contribute to the development of diseases, including various types of cancer. ${ }^{3,4}$ Further research on the mechanisms by which the gut microbiota influences the occurrence and development of cancer is warranted. Nevertheless, available evidence suggests that changes in the microbial composition of the specific gut microbiota increase host cell mutagenesis. This is achieved either by influencing metabolism and/or immunity to create an immunosuppressive environment that promotes cancer, or through an inflammatory cascade that leads to the initiation and progression of cancer.

The development of a precursor disease into cancer often requires years. Hence, screening and early identification are key to preventing the progression of disease. Evidence has shown that changes in the gut microbiome that occur in the early 
stages of colorectal cancer (CRC) may be used to identify individuals at risk of developing colorectal adenoma (a precursor lesion of CRC). ${ }^{5}$ Therefore, changes in the gut microbiome may serve as biomarkers for precancerous lesions or the early detection of cancer. Moreover, the gut microbiome can influence the efficacy or toxicity of a variety of treatments, including immunotherapy. ${ }^{6}$ Researchers are conducting clinical trials to improve the outcomes of cancer treatment by manipulating the gut microbiome. Therefore, the study of changes in the intestinal microbiota is of great importance for the evaluation of human health status, in-depth study of the pathogenesis of cancer, and search for new therapeutic targets and drugs. At present, there have been some reviews on the relationship between microbes and cancer, with varying emphasis. Garrett retrospected how the microbiome affects cancer, responsiveness to cancer treatments, and cancer-related complications. ${ }^{7}$ Helmink et al mainly reviewed the effects of the gut microbiota on cancer development and treatment, ${ }^{8}$ while Gopalakrishnan and colleagues focused on immunity. ${ }^{9}$ This review summarizes the latest research progress on the gut microbiota and cancer in a comprehensive way, which includes mechanisms, treatments, biomarkers, interventions, clinical trials and so on, hoping to help researchers and clinicians develop new cancer diagnosis and treatment strategies.

\section{Gut Microbiota in a Healthy State Gut Microbiota}

The microbial community in the gastrointestinal ecosystem is termed the gut microbiota. ${ }^{10}$ The gut microbiota consists of $>10 \times 10^{14}$ microbes, including bacteria, viruses, fungi, and archaea. Bacteria constitute the majority of the gut microbiota; the dominant species are fairly stable, representing four main phyla, namely Bacteroidetes, Firmicutes, Actinobacteria, and Proteobacteria. ${ }^{11}$ Notably, there are significant differences in bacterial composition between the small intestine and the colon. Due to host features such as mucus, $\mathrm{pH}$, bile acids, regional oxygen levels, gastrointestinal transport time, and immune factors, as well as microbial community dynamics, the diversity and abundance of the gut microbiota normally increases from proximal to distal. The jejunal microflora is estimated to be $10^{4}-10^{7} \mathrm{CFU} / \mathrm{mL}$, and is mainly represented by Firmicutes, but also includes Actinobacteria, Proteobacteria, and Bacteroidetes. The ileum microbiota is mainly a facultative and compulsory anaerobes that include Bacteroides, Enterobacteria, Clostridium, Enterococcus, Veillonella, and Lactobacillus. The microbial load is approximately $10^{3}-10^{8} \mathrm{CFU} / \mathrm{mL}$. Colonic microbiota can reach $10^{10}-10^{12} \mathrm{CFU} / \mathrm{mL}$ and includes Firmicutes, Bacteroidetes, Lachnospiraceae, Bacteroidaceae, and Prevotellaceae, as well as strict colonic anaerobes, such as Eubacteria, Clostridium, and Roseburia. ${ }^{12-14}$ Overall, the structure of the gut microbiota is determined by host genetics, initial microbial exposure, sex, diet, environmental factors, stress, and disease. ${ }^{15,16}$ Polysaccharides (in the form of plant fibers) and their metabolites and short-chain fatty acids (SCFAs) have a positive influence on the of the gut microbiota. ${ }^{17,18}$

\section{A Balanced Gut Microbiota is Critical for the Host}

The establishment of an intestinal microbial ecosystem consisting of a variety of native symbiotic species is crucial for the host. ${ }^{19}$ Colonization of the intestine by microorganisms synchronizes with maturation of the immune system and plays a fundamental role in the induction, training, regulation, and function of the host immune system. ${ }^{20}$ Throughout the lifetime of an individual, the peaceful co-existence of intestinal symbiont microorganisms is destroyed, leading to serious immune deficiency and increased risk of disease. ${ }^{21}$ Moreover, the gut microbiota regulates a series of processes at the cellular and molecular levels, leading to the maturation, differentiation, and proliferation of the intestinal mucosa. ${ }^{22}$ Interestingly, studies have determined that the gut microbes also play a significant role in processes of neurogenesis, such as blood-brain barrier formation, myelination, neurogenesis, neurotransmitter production, and microglial cell maturation, as well as regulating numerous aspects of animal behavior. ${ }^{23,24}$ Lactobacillus and Bifidobacterium produce the main inhibitory neurotransmitter, aminobutyric acid, by metabolizing glutamate, the most abundant free amino acid and excitatory neurotransmitter in the brain. ${ }^{25}$

Gut microbiota can also regulate host physiological functions by metabolizing dietary components. Butyric acid is mainly generated by Faecalibacterium prausnitzii (F. prausnitzii), Roseburia intestinalis, Eubacterium rectale, and Roseburia spp. by fermentation of dietary fiber. ${ }^{26}$ It provides energy to colon cells, exhibits antiinflammatory properties, induces cell differentiation and apoptosis of cancer cells, protects histone hyperacetylation activity, and inhibits angiogenesis. ${ }^{27,28}$ Biogenic amines 
are another class of metabolites produced by gut microbes through the decarboxylation of dietary amino acids. These metabolites play a role in DNA stabilization and act as precursors of hormones, alkaloids, proteins, and nucleic acids. ${ }^{29}$ Furthermore, the gut microbiota is a major donor of acetyl groups in acetylation reactions, responsible for regulating gene expression. ${ }^{27}$

The human gut microbiota can synthesize at least eight B vitamins. Vitamin B12, which has a complex structure, is produced only through bacterial fermentation, while the absorption of vitamin $\mathrm{K}$ also requires the transformation of intestinal bacteria. ${ }^{30}$

In general, the gut microbiota can metabolize indigestible ingredients in food, synthesize nutrients such as vitamins, control energy homeostasis, detoxify metabolites, regulate immune responses, promote the development of the nervous system, provide signals for epithelial cell renewal and maintenance of mucosal integrity, and secrete antibacterial products. $^{18}$

\section{Dysbiosis and Cancer}

A balanced gut microbiota benefits host health, while ecological imbalance promotes the development of diseases, including cancer. ${ }^{3,31}$ Dysbiosis can be caused by a variety of factors, such as diet, antibiotics and stress. ${ }^{32}$ It is usually manifested by loss of symbiosis, pathogen proliferation, and/or reduction in alpha diversity, resulting in a shift of the metabolome to an inflammatory state conducive to carcinogenesis. ${ }^{32,33}$ Moreover, dysbiosis renders individuals vulnerable to opportunistic pathogens that can release toxins, resulting in genomic instability and potentially carcinogenic effects. ${ }^{34,35}$ It has been confirmed that Streptococcus bovis, Helicobacter pylori, Fusobacterium nucleatum (F. nucleatum), and Enterococcus faecalis (E. faecalis) are related to cancer. $^{36-38}$

\section{Gut Microbiota in the Context of Cancer}

Notably, researchers have reported significant changes in gut microbes in various types of cancer. Jing et al reported that, compared with healthy controls, the proportions of Firmicutes and Proteobacteria were increased in patients with thyroid carcinoma, whereas that of Bacteroidetes was decreased. At the genus level, the abundance of Bacteroides megamonas and Trichoricaceae genera was decreased in patients with thyroid carcinoma. ${ }^{39}$ Goedert et al reported that the alpha diversity of the gut microbiota in postmenopausal women with breast cancer was lower than that observed in pairs of healthy controls. Moreover, the relative abundance of Clostridiaceae, Faecalibacterium and Ruminococcaceae was relatively high, whereas that of Dorea and Lachnospiraceae was relatively low. ${ }^{40}$ Vernocchi et al observed that numbers of symbiotic bacteria, such as Akkermansia muciniphila, Rikenellaceae, Bacteroides, Peptostreptococcaceae, Mogibacteriaceae, and Clostridiaceae were reduced in patients with non-small cell lung cancer. ${ }^{41}$ In another cohort study of early-stage lung cancer, Proteobacteria, including many harmful microorganisms, were significantly enriched in cancer groups. Firmicutes and actinobacteria, which promote the production of short-chain fatty acids and regulate inflammation and tumorigenesis, were dramatically reduced. At the genus level, Ruminococcus, an uncharacterized genus of Lachnospiraceae and an uncharacterized genus of Enterobacteriaceae were obviously increased in the cancer group, while Veillonella, Faecalibacterium, Bifidobacterium, and Streptococcus were markedly enriched in the healthy controls. $^{42}$

In previous gastric cancer (GC) studies, Peptostreptococcus stomatis, Dialister pneumosintes, Streptococcus anginosus, Parvimonas micra (P. micra), Slackia exigua, Clostridium colicanis, and F. nucleatum were significantly enriched, whereas Helicobacterium was depleted. $^{43-45}$ A recent study showed that GC patients presented notably different gut microbiota from healthy controls. At the phylum level, Chloroflexi, TM7, Acidobacteria, Actinobacteria, Verrucomicrobia, Fusobacteria, Nitrospirae, and Planctomycetes increased in the stool of GC patients. At the genus level, 27 genera were enriched, including Leptotrichia, Fusobacterium, Lactococcus, Prevotella, and Porphyromonas, while Megamonas decreased. ${ }^{46}$ Interestingly, comparing GC and $\mathrm{CRC}$, there was no remarkable difference in the bacterial diversity between the cancer types. Among the 8 phyla enriched in GC, 5 phyla were enriched in $\mathrm{CRC}, 25$ of the 28 genera showed the same trend. ${ }^{46}$ These results indicated that the overlap of dysbiosis in different cancers may be a common basis for the occurrence and development of various cancers, which points out a new direction for further research on the mechanism of cancer. Proteobacteria, Synergistetes, and Euryarchaeota were abundant in the intestines of patients with pancreatic ductal adenocarcinoma (PDAC). Proteobacteria accounted for approximately $50 \%$ and $8 \%$ of the gut microbiota of patients and healthy controls, respectively. ${ }^{47}$ 
In a study of $52 \mathrm{CRC}$ patients and 55 healthy family members, metagenomic sequencing showed that compared with controls, Coprobacillus, Peptoniphilus, Burkholderia, Paracoccus, Synechococcus, Porphyromonas, and Cyanothece were significantly enriched in colorectal cancer patients. At the species level, patients accumulated Clostridium ramosum, Roseburia inulinivorans, Porphyromonas gingivalis, Gemella morbillorum, and F. nucleatum. In addition, microbial genes were also reduced in CRC patients, with 624,404 genes found in the control group and only 585,092 genes found in CRC. The proportion of amino acid metabolizing bacteria in the gut microbiota of patients decreased. ${ }^{48}$ In 2019, the University of Trento analyzed five open datasets and two new cohorts, totaling 969 fecal metagenomes, and found that the gut microbiota of CRC was more abundant than that of the control group, partly because of the expansion of oral microbes. F. nucleatum, Porphyromonas asaccharolytica, Solobacterium moorei, Peptostreptococcus stomatis, Parvimonas micra, Clostridium symbiosum, Streptococcus dysgalactiae, Streptococcus tigurinus, Streptococcus gallolyticus, and Gemella morbillorum were enriched in patients, while Bifidobacterium catenulatum and Gordonibacter pamelae were enriched in the healthy control group. ${ }^{49}$ Kostic et al compared the enrichment of $F$. nucleatum in the colonic tumor-related microbiota of patients with CRC compared with levels recorded in adjacent normal mucosa. ${ }^{50}$ Other researchers found that the colonization rate of $F$. nucleatum in the lumen of patients with colorectal adenoma was higher than that observed in healthy controls. Furthermore, the colonization of F. nucleatum in adenoma was increased compared with that noted in adjacent normal mucosa. ${ }^{51}$ These data strongly suggest that $F$. nucleatum is associated with CRC and may play a role in the early stages of disease. ${ }^{52}$ Another study involving patients with CRC showed an increase in $F$. nucleatum and Campylobacter and a decrease in butyrate-producing bacteria in fecal samples. ${ }^{53}$

\section{The Mechanism of Gut Microbiota Promoting the Development of Cancer}

The occurrence and development of cancer is a complex process involving genetic mutations, the tumor microenvironment, and inflammatory mediators, in which gut microbes play an important role. Herein, we present the available evidence and discuss the mechanisms involving microorganisms causing cancer from the aspects of metabolism, immunity, inflammation, etc. Various mechanisms interact and form a network to jointly promote the occurrence and development of cancer. Broadly speaking, mainly by affecting host metabolism, cell proliferation, inflammatory response, and the immune status, the gut microbiota regulates cancers in terms of susceptibility to genetic instability, initiation, progression of immune responses, comorbidities, and response to treatment. ${ }^{3,54}$

\section{Increase in Host Cell Mutagenesis}

At present, it is known that the gut microbiota produces genotoxins, free radicals, and reactive oxygen species (ROS) that can damage host DNA, alter cell cycle control, accelerate cell proliferation, and disrupt the normal process of controlled cell death, thereby increasing the risk of cancer. ${ }^{55}$ For example, Helicobacter pylori colonizing the gastric mucosa produces a cytotoxin-associated gene A (CagA) oncoprotein, which leads to reprogramming of gastric epithelial cells, thus participating in the pathogenesis of gastric cancer. ${ }^{37}$ Porphyromonas gingivalis secretes peptide-arginine deaminase that may induce p53 and KRAS point mutations, which are major genetic drivers of pancreatic cancer. ${ }^{56}$ Colibactin produced by polyketide synthetase-positive Escherichia coli (E. coli) and Enterobacteriaceae breaks the double-strand DNA of host cells, while Porphyromonas spp produce ROS that damage the host DNA. ${ }^{57,58}$ Direct interactions of bacterial structural components and their metabolites (eg, hydrogen sulfide and para-cresol) with epithelial mesenchymal cells and hematopoietic cells may exert direct genotoxic effects and promote cancer progression. ${ }^{59,60}$ Numerous microbes, including B. fragilis, F. nucleatum, and E. faecalis, produce toxins that alter the normal adhesion between cells, thereby facilitating the transformation of resting epithelial cells into moving mesenchymal cells. Of note, epithelial-to -mesenchymal transformation is a key step in the transformation of benign tumors to malignant tumors. ${ }^{55}$ F. nucleatum is often enriched in patients with CRC and is related to DNA methylation in inflammatory colon mucosa. ${ }^{61}$

\section{Promotion and Maintenance of a Pro-Inflammatory Environment}

It is well-established that inflammation is involved in carcinogenesis through mutations, genomic instability, 
and epigenetic modifications. ${ }^{62}$ For instance, chronic inflammation caused by infection with Helicobacter pylori leads to abnormal DNA methylation in gastric mucosa and activation-induced cytidine deaminase through the activation of nuclear factor- $\kappa \mathrm{B}(\mathrm{NF}-\kappa \mathrm{B})$ in gastric epithelial cells, thereby leading to mutations. ${ }^{63} \mathrm{~A}$ large number of studies have shown that the gut microbiota plays an important role in the inflammatory response. For instance, Lactobacillus, Proteobacteria, Clostridium difficile, Enterococci, and B. fragilis, can impact on different immune cells and play pro- and anti-inflammatory roles. $^{64}$ Microorganisms activate the inflammatory response, increase the recruitment of pro-inflammatory cells, and the secretion of cytokines, enhance oxidative stress, change energy dynamics, and lead to DNA damage. These effects eventually result in molecular changes and tumor transformation, as well as promote tumor growth, invasion, and metastasis. ${ }^{65}$ Liam et al colonized APC $^{\text {Min }}$ mice with enterotoxigenic B. fragilis (ETBF) and found that the $B$. fragilis toxin (BFT) triggered a multistep inflammatory cascade in colic epithelial cells (CECs). ${ }^{35}$ This cascade is required to promote carcinogenesis through the interleukin-17 receptor (IL-17R), NF- $\mathrm{BB}$, and STAT3 signaling pathways. Notably, the activation of IL17-dependent NF- $\mathrm{KB}$ in CECs induces proximal-to-distal mucosal gradients of $\mathrm{C}-\mathrm{X}-\mathrm{C}$ chemokines, including $\mathrm{C}-\mathrm{X}-\mathrm{C}$ motif chemokine ligand 1 (CXCL1). ${ }^{66}$ This mediates the recruitment of immature myeloid cells expressing the $\mathrm{C}-\mathrm{X}-\mathrm{C}$ motif chemokine receptor 2 (CXCR2) and is parallel to ETBF-mediated distal colonic tumorigenesis. ${ }^{66}$ Thus, BFT induces pre-oncogenic signaling from CEC to the mucosal T-helper 17 (Th17) response and selectively activates $\mathrm{NF}-\kappa \mathrm{B}$ in the distal colon CECs, which together trigger myeloid cell-dependent distal colon tumorigenesis. $^{66}$

Using a hormone receptor-positive $(\mathrm{HR}+)$ breast cancer mouse model, Buchta Rosean et al found that a preestablished symbiotic disorder leads to enhanced tumor cell spread, and that the symbiotic disorder leads to increased inflammation and infiltration of myeloid cells in the breast. ${ }^{67}$ These results suggest that the symbiotic disorder has a sustained effect on the spread of HR+ breast cancer, and that the increased spread in mice with symbiotic disorder is independent of tumor growth dynamics. ${ }^{67}$ Moreover, this evidence demonstrated that dysbiosis is a pre-existing host-intrinsic regulator of tissue inflammation, myeloid recruitment, fibrosis, and tumor cell proliferation in $\mathrm{HR}+$ breast cancer. $^{67}$
Intestinal bacteria can also upregulate the levels of tolllike receptor (TLR), activate the cancer-related inflammatory signaling pathway NF- $\kappa \mathrm{B}$, lead to the release of IL-6, IL-12, IL-17, IL-18, and tumor necrosis factor (TNF) $\alpha$, to trigger persistent inflammation in the cancer microenvironment, which is vital in the regulation of inflammation and cancer-related processes. ${ }^{68,69}$ In a cancer setting, inflammatory markers are responsible for cell proliferation, invasion, angiogenesis, and suppression of certain immune functions. $^{70}$

\section{Disruption of Immune Stability}

Under pathological conditions, when the intestinal microbiota is disturbed or the intestinal mucosal barrier is disrupted, microbial-related molecules stimulate macrophages and dendritic cells to produce proinflammatory cytokines. Subsequently, these cytokines activate adaptive immune cells and lead to the disruption of immune stability. ${ }^{71}$ Dysbiosis may also lead to the inappropriate release of cytokines (eg, IL-17 and IL-22) from innate lymphoid cells, resulting in chronic inflammation and susceptibility to cancer. Furthermore, abnormal innate lymphoid cell responses to dysbiosis may also influence $\mathrm{T}$ cell responses, further promoting chronic inflammation and cancer. ${ }^{72}$

F. nucleatum stimulates anti-inflammatory myeloid cells, interferes with natural-killer and $\mathrm{T}$ cells functions by activating $\mathrm{T}$ cell immunoreceptor with $\mathrm{Ig}$ and ITIM domains (TIGIT) and CEA cell adhesion molecule 1 (CEACAM1) inhibitory receptors, and induces Wnt/catenin (catenin beta 1) modulator annexin A1, thereby creating a tumor-promoting immunosuppressive environment. This process leads to the initiation and progression of CRC. ${ }^{73-75}$ B. fragilis drives the differentiation of IL10secreting regulatory $\mathrm{T}$ (Treg) cells. Treg cells impair anticancer Th1 immunity and participate in the progression and aggressiveness of gliomas. ${ }^{70}$ The gut microbiota can also induce the expression of immunosuppressive chemokines in liver cells, leading to the accumulation of myeloid-derived suppressor cells (MDSCs) and ultimately promoting the development and growth of cholangiocarcinoma. $^{76}$ Molecular patterns associated with pathogenic microorganisms are also recognized by innate immune system cells through pattern recognition receptors, including TLRs and NOD-like receptors, chronic activation of TLRs, promotion of cancer cell proliferation, and an increase in invasion and metastasis by regulating cytokines, metalloproteinases, and pro-inflammatory 
integrins. ${ }^{77}$ For example, in the early stages of pancreatic tumors and in established PDAC, microbial-induced TLR activation suppresses both the innate and adaptive immunity of the host. ${ }^{78,79}$ Specifically, in addition to inducing the transformation of pancreatic cancer stellate cells into fibrocytes, TLR9 also attracts immunosuppressive Treg cells and MDSCs into the tumor environment. ${ }^{80}$ Lipopolysaccharide and TLR4 ligation through dendritic cell-dependent Th2 immune response aggravates pancreatic inflammation and expedites the development of pancreatic tumors. Microbial-mediated ligation of TLR2 and TLR5 limits T-cell-mediated immunity by inducing a macrophage immunosuppressive phenotype. ${ }^{28}$

\section{Metabolic Changes}

Gut microorganisms are involved in a series of metabolic activities of the host, and dysbiosis may alter the expression of lipid metabolism-related microRNAs, leading to obesity and cancer. ${ }^{81}$ It has been hypothesized that dysregulation of the microbiome and microRNAs may be involved in the pathogenesis of cancer in the central nervous system through the microbial-enteric-brain axis. ${ }^{82}$ Furthermore, during cancer development, gut microbes may undergo metabolic reprogramming. For example, Zheng et al in a study of early non-small cell lung cancer found that 19 of 328 metabolic pathways detected were enriched in the cancer group, including steroid biosynthesis, cell antigens, transcription-related proteins, the ubiquitin system, and bile secretion. However, 12 pathways related to bacterial chemotaxis, G protein-coupled receptors, bacterial motility proteins, flavone, flavonol biosynthesis and apoptosis were all reduced. ${ }^{42}$ Microbial metabolites or co-metabolites produced by host and microbial contributions can cause inflammation and affect the balance of cell proliferation and death in tissues. ${ }^{83}$ Several bacterial metabolites (eg, SCFAs or secondary bile acids) and microbial-related molecular patterns (eg, lipopolysaccharides and peptidoglycan) influence host nutrient uptake, metabolism, intestinal barrier, and systemic inflammatory responses. $^{84}$ For example, members of Clostridium bacteria XI and XIVa convert primary bile acids (deoxycholic acid and cholic acid) into secondary bile acids (lithocholic acid and deoxycholic acid), which exert potential DNA damage and carcinogenic effects. ${ }^{85}$ Lithocholic acid and deoxycholic acid, trigger colon cancer development through the regulation of M3R and Wnt/ $\beta$-catenin signaling in order to convert normal colon epithelial cells convert into CSC. ${ }^{86}$ Clostridium IV and XIVa, which include the genera Eubacteria, Rosa, and Cofaecium, metabolize dietary fiber and polysaccharides in the colon to produce acetic acid, which is converted to acetyl-coenzyme A (acetylCoA) by acetylCoA synthetase short-chain family member 2 expressed by cancer cells. ${ }^{87}$ This process stimulates the anabolic response of cancer cells and supports the growth of numerous types of cancer, including glioblastoma, breast cancer, ovarian cancer, and lung cancer. $^{88-90}$

Hydrogen sulfide $\left(\mathrm{H}_{2} \mathrm{~S}\right)$ is mainly produced by the colo-intestinal bacteria, such as E. coli and Salmonella, by degrading sulfur-containing amino acids. Studies have reported the anti-inflammatory activity of $\mathrm{H}_{2} \mathrm{~S}$ in the gut, while others have shown harmful effects, suggesting that these results may be related to the environment. ${ }^{91} \mathrm{H}_{2}$ $\mathrm{S}$ exhibits deleterious reactions in intestinal epithelial cells. Relative levels of $\mathrm{H}_{2} \mathrm{~S}$ in the colon directly regulate oxidative phosphorylation in epithelial cells and elevated $\mathrm{H}_{2} \mathrm{~S}$ levels inhibit the electron transport chain complex IV. ${ }^{92}$ In addition, $\mathrm{H}_{2} \mathrm{~S}$ induces genotoxic damage in epithelial cells, inhibits the metabolism of SCFAs, and induces rupture of the mucous barrier, thereby exposing the contents of the lumen to the underlying tissues. ${ }^{93}$

A better understanding of the mechanism through which specific microbial pathogens cause specific carcinogenic effects may lead to the discovery of valuable biomarkers for the diagnosis and treatment of cancer.

\section{Biomarkers}

Screening and early identification of cancer correlates with patient outcomes. For example, the incidence and mortality of colorectal cancer can be significantly reduced by screening for precancerous lesions such as adenomatous polyps or early colorectal cancer and appropriate treatment, and the 5-year relative survival rate can reach about $90 \%{ }^{94,95}$ As mentioned earlier, significantly different gut microbiota have been observed in patients with various types of cancer versus healthy individuals, and these differences may permit the use of specific bacteria as biomarkers.

In one study, bacterial DNA was extracted from the feces of 31 patients with early-stage breast cancer. Realtime quantitative polymerase chain reaction was used to amplify bacterial community specific $16 \mathrm{~S}$ rRNA gene sequences. The results showed that the percentages and absolute numbers of Clostridium coccoides, $F$. prausnitzii, and Blautia differed significantly according to the clinical staging and tissue prognosis grading. ${ }^{96}$ Among patients 
with different histoprognostic grades of breast cancers, the abundance of Blautia spp. bacteria increased markedly in parallel with grade. The total numbers of Bacteroidetes, Clostridium coccoides cluster, Clostridium leptum cluster, F. prausnitzii, and Blautia sp. were significantly higher in clinical stage groups II/III than at clinical stages 0/I, with higher percentages observed for Clostridium leptum cluster. $^{30}$

Jia et al found that patients with intrahepatic cholangiocarcinoma (ICC) had the highest $\alpha$-diversity and $\beta$ diversity of intestinal flora and increased abundance of Lactobacillus, Actinomyces, Peptostreptococcaceae, and Alloscardovia versus those with hepatocellular carcinoma or cirrhosis and healthy individuals. ${ }^{97}$ The plasma-fecal ratios of glycoursodeoxycholic acid and tauroursodeoxycholic acid (TUDCA) were significantly increased in patients with ICC. Moreover, the combination of Lactobacillus and Alloscardovia was positively correlated with the plasma-fecal ratio of TUDCA, which could distinguish ICC from hepatocellular carcinoma, liver cirrhosis, and healthy individuals. ${ }^{97}$ Vascular invasion (VI) often leads to poor prognosis in patients with ICC. Compared with ICC patients without VI, those with VI had a richer Ruminococcaceae family, increased plasma levels of IL-4 and decreased plasma levels of IL-6 and chenodeoxycholic acid. In patients with ICC, the plasma levels of taurocholic acid were positively correlated with those of IL-4. In two mouse tumor models, plasma TUDCA was inversely associated with the abundance of Pseudoramibacter and survival in patients with ICC; however, it had no effect on tumor size. $^{97}$

In a case-control study of $\mathrm{CRC}$, polymerase chain reaction was performed on fecal samples obtained from 60 patients and 60 healthy volunteers using neu and BFT (BFT-1, BFT-2, and BFT-3 are enterotoxin isotypes) as marker genes. ${ }^{98}$ The frequency of $B$. fragilis in the CRC and control groups was $58.3 \%$ and $26.6 \%$, respectively. The detection rate of the BFT gene in patients with CRC was significantly higher than that observed in the control group. Furthermore, the presence of the BFT gene was significantly higher in patients with CRC stage III than in those with stage II. ${ }^{98}$ The detection rate of the enterotoxin isotype BFT-2 was higher in patients with CRC versus healthy controls. This evidence suggested that the detection of ETBF may be a potential marker for the diagnosis of CRC. ${ }^{98}$

In two recent cohort studies, Löwenmark et al found that the abundance of $P$. micra was significantly higher in fecal samples obtained from patients with CRC than in those collected from controls. ${ }^{99}$ In the Faecal and Endoscopic Colorectal Study in Umeå, Sweden (FECSU) cohort, the sensitivity and specificity of $P$. micra in feces for the detection of cancer were $60.5 \%$ and $87.3 \%$, respectively. In the U-CAN cohort, these values were $56.7 \%$ and 92.6\% , respectively. ${ }^{99}$ Moreover, added microbial markers F. nucleatum, clbA + bacteria, and fecal hemoglobin enhanced the sensitivity of the assay; nevertheless, the specificity was reduced. Therefore, $P$. micra, as a candidate microbial marker for non-invasive screening, had the potential to improve diagnostic performance. ${ }^{99}$

\section{Clinical Application of Microorganisms}

In 1928, Fleming accidentally discovered penicillin (a product of mold that has antibacterial effects) in an experiment involving Staphylococcus bacteria. ${ }^{100}$ This discovery introduced a new era of using microbes against disease. With the development of science and technology, the use of microorganisms or their metabolites to treat diseases was established. For example, fecal microbiota transplantation (FMT) is a common treatment for recurrent Clostridium difficile colitis with a good safety profile. ${ }^{101}$ Intrabladder injection of Bacillus Calmette-Guerin has also become the standard treatment for moderate- to highrisk non-muscularly invasive bladder cancer. ${ }^{102}$ In the following section, we focus on the influence of gut microbiota on the efficacy of treatment against cancer and its utility in this setting.

\section{Gut Microbes Influence the Treatment of Cancer}

In the course of cancer treatment, the gut microbiota regulates the efficacy and toxicity of chemotherapy through various mechanisms, such as translocation, immune regulation, metabolism, enzyme degradation, and diversity reduction; these mechanisms are collectively referred to as the timer mechanism framework. ${ }^{103}$ For example, cyclophosphamide damages the intestinal barrier and increases intestinal permeability. Enterococcus hirae is transferred from the small intestine to secondary lymphatic organs and increases the intratumoral CD8/Treg ratio. ${ }^{104}$ Barnesiella testinihominis accumulates in the colon, increases the number of INF- $\gamma$-producing $\gamma \delta$ Tcells in the tumor bed, shifts the immune system to a proinflammatory state, and synergically enhances the efficacy 
of cyclophosphamide. ${ }^{104}$ The Th17 response is reduced in sterile or antibiotic-treated tumor-bearing mice with cancers resistant to cyclophosphamide. ${ }^{105}$ Oxaliplatin exerts its anti-cancer activity through ROS. Intestinal microorganisms stimulate bone marrow cells to produce ROS. ${ }^{106}$ In the absence of intestinal microorganism involvement, the production of microbial-dependent ROS is reduced, eliminating the cytotoxicity of oxaliplatin in mice and preventing the killing of cancer cells. ${ }^{107}$ Moreover, $F$. nucleatum promotes resistance to oxaliplatin and 5-fluorouracil (5-FU) by coordinating TLR to activate miRNA expression and the autophagy network. ${ }^{38}$ Bacteroides and Clostridium, which produce $\beta$ glucosidase, are associated with the accumulation of diarrhea-inducing metabolites induced by irinotecan and 5-FU chemotherapy, while Raoultella planticola effectively inactivates doxorubicin by it deglycosylation to 7-deoxydoxorubicinol and 7- deoxydoxorubicinolone under anaerobic conditions. ${ }^{108,109}$ Inactivation of gemcitabine, a chemotherapy drug for PDAC, depends on the expression of a specific subtype of the bacterial enzyme cytidine deaminase, which is common in gamma-proteobacteria. ${ }^{110}$

In addition to chemotherapy, the gut microbes also influence the effectiveness of radiation therapy. Researchers used vancomycin to treat mouse models of melanoma and lung cancer expressing the E6/7 of human papillomavirus and cervical cancer. The changes in grampositive intestinal flora reshaped the tumor microenvironment, increased the antigen presentation of draining lymph nodes, and improved the anti-tumor effect of radiotherapy. ${ }^{111}$ Indole-3-propionic acid (IPA) is derived from intestinal microorganisms and is a tryptophan deamination product with intracellular signaling activity. ${ }^{112}$ Xiao et al found that IPA exerted a radio-protective effect on mice, which was attributed to the lower level of systemic inflammation, reduced myelosuppression, restoration of hematopoietic organ function, and improvement of gastrointestinal function and epithelial integrity in mice treated with IPA after irradiation. ${ }^{113}$ Ferreira et al reported that enteropathy in patients who received pelvic radiotherapy was related to the composition of intestinal microbes, with increased abundance of Clostridium, Rosesporium, and Phascolarctobacterium in patients who had toxic reactions. ${ }^{114}$

The application of immune checkpoint inhibitors represents an important advance in cancer therapy. Immune checkpoint inhibitors, which bind to immune checkpoint proteins to relieve tumor-induced inhibition of $\mathrm{T}$ cell function, have been approved for the treatment of a variety of malignancies, including melanoma, lung cancer, stomach cancer, Hodgkin's lymphoma, ovarian cancer, and more. ${ }^{115}$ Studies using mouse tumor models have shown that the gut microbiota composition is vital for promoting anti-tumor immune responses to anticytotoxic T-lymphocyte-associated protein 4 (antiCTLA-4) and anti-programmed cell death-ligand 1 (antiPD-L1) monoclonal antibodies. ${ }^{116,117}$ Several mouse models of melanoma have shown that the effectiveness of programmed cell death protein 1 (PD-1) inhibitors is reduced under aseptic conditions and increased in the presence of Bifidobacterium. ${ }^{118}$ This activates antigenpresenting cells, thereby promoting the accumulation of activated $\mathrm{CD} 8+\mathrm{T}$ cells in the tumor microenvironment. ${ }^{117}$ The efficacy of PD-1/PD-L1 blockade in non-small cell lung cancer, renal cell carcinoma, and urothelial carcinoma was partially offset by bacterial ablation. ${ }^{119}$ Another study found that antibioticinduced dysbiosis was associated with a reduced PD-1 blocking effect and poor clinical efficacy. The survival time of anti-PD-1 monoclonal antibodies was positively correlated with the relative abundance of Akkermansia, one of the most abundant bacteria in the ileum of healthy individuals. ${ }^{120}$ In addition, transfer of intestinal microbes in patients with cancer who had responded to immunotherapy and oral supplementation of Akkermansia improved the efficacy of immunotherapy. ${ }^{120}$ Furthermore, in a study of tumor-bearing mice, bacterial ablation significantly reduced the therapeutic efficacy of CTLA-4 blocking, while the use of B. fragilis showed potential to overcome resistance to anti-CTLA-4 immunotherapy in germ-free mice. ${ }^{120}$

A study of 39 patients with metastatic melanoma who underwent immune checkpoint therapy also showed a significant correlation between the microbial content and response to immunotherapy. ${ }^{121}$ Bacteroides thetaiotaomicron, F. prausnitzii, and Holdemania filiformis were abundant in the intestines of responders to cancer immunotherapy. ${ }^{50}$ Transfer of the feces of patients with melanoma into mice demonstrated that FMT could improve the effectiveness of immunotherapy and, thus, optimize existing therapies. ${ }^{118}$

With further research into the mechanisms of these relationships, the composition of the gut microbiota is a potential biomarker for predicting individual therapeutic outcomes and a target for improving these outcomes. ${ }^{122}$ 


\section{Manipulation of Gut Microbes to Interfere with Cancer}

Scientists have proposed several ways to take advantage of gut microbes for the prevention and treatment of cancer, as well as the mitigation of the toxic side effects of chemotherapy and radiotherapy. These approaches to achieving better clinical outcomes for patients with cancer undergoing immunotherapy include dietary modifications and use of probiotics, selected antibiotics, and FMT (Table 1).

Studies have shown that a reduction in the consumption of animal fat reduced the abundance of harmful Bacteroidetes species, while a high-fiber diet increased the number of microorganisms producing SCFAs, such as Eubacterium rectale, Roseburia species, and F. prausnitzii. ${ }^{123}$ High consumption of whole grains was associated with an increase in the number of SCFAproducing microorganisms (eg, Roseburia, Lachnospira) and a decrease in pro-inflammatory microorganisms (eg, Enterobacteriaceae). Consumption of fermented foods may also contribute to a protective metabolic environment due to their probiotic content, particularly Lactobacillus casei CRL431. ${ }^{124}$

Probiotics can prevent the proliferation of pathogenic bacteria, regulate gut microbiota and metabolism, maintain the integrity of intestinal barrier, reduce intestinal inflammation, enhance immune response, bind or inactivate carcinogens, so as to protect against tumors. ${ }^{125,126}$ For example, Lactobacillus acidophilus and Bifidobacterium bifidum both strengthen the intestinal epithelial tight junction barrier and prevent against intestinal inflammation, and the former was induced in TLR-2 dependent and a strain-specific manner, while the latter was NF- $\kappa \mathrm{B}$ independent in targeting the TLR-2 pathway. ${ }^{127,128}$ In addition, Parisa et al using in vivo and in vitro studies that bifidobacterium inhibits CRC by down-regulating HER-2, EGFR, and PTGS-2 (COX-2). ${ }^{129}$ Chou et al used azoxymethane/dextran sulfate sodium to induce colitisassociated CRC in ICR mice. After 14 weeks of treatment with Lactobacillus fermentum V3 (1'108 CFU/d) (5 days/ week, once a day), Compared with the control group, the levels of lactobacillus in feces were significantly increased, and the abundance of harmful bacteria, Bacteroides and Akkermansia, was markedly decreased. Pro-inflammatory factors such as IL- $1 \alpha$, IL-1 $\beta$, and IL-6 were dramatically reduced. The infiltration of CD68+ macrophages in tumors was reduced, and tumor growth was observably inhibited. ${ }^{130}$ More recently, Chung et al synthesized stable synthetic probiotics using Pediococcus pentosaceus and $\mathrm{P} 8$ therapy protein that ameliorated azoxyMethane and Dextran sodium sulfate-induced colitis-associated CRC impaired flora, while tumor growth and tumor volume were significantly inhibited. ${ }^{131}$ Furthermore, Lactobacillus kefiri LKF01 is safe and effective in preventing severe diarrhea in cancer patients receiving 5-FU or capecitabine-based treatment. ${ }^{132}$

Prebiotics are usually fibers or polyphenols that cannot be digested by the host and are selectively used by gut microbes to produce health benefits. ${ }^{133}$ Common prebiotics include fructose oligosaccharide, inulin, and galactose oligosaccharide, which in high doses can increase the abundance of Lactobacillus and Bifidobacterium. ${ }^{133}$ In a mouse model of melanoma, supplementation with inulin or mucin enhanced anti-tumor immune activity through significant changes in intestinal flora, thereby inhibiting tumor growth. ${ }^{134}$ In addition, inulin may limit the growth of colon tumors. ${ }^{29}$ In mice fed with inulin, the growth of colon cancer was inhibited and Akkermansia muciniphila was significantly enriched. Moreover, Akkermansia muciniphila was also associated with a therapeutic response to anti-PD-1/PD-1 immunotherapy. ${ }^{29,120}$

Intriguingly, studies have shown that exercise can independently alter the gut microbiome. Among premenopausal women, those performing $150 \mathrm{~min}$ of moderate aerobic exercise per week (in line with the recommendations of the World Health Organization) had higher levels of Akkermansia muciniphila, F. prausnitzii, and Roseburia hominis than those who were sedentary. These species play health-promoting roles, such as maintaining the intestinal barrier. $^{135}$

In FMT, the functional flora in the feces of healthy individuals is transplanted into the gastrointestinal tract of patients to reconstruct the new intestinal flora and treat intestinal and extraenteral diseases. As an effective means for reconstructing the intestinal flora, FMT has been used in the treatment of and exploratory research on infection with Clostridium difficile, inflammatory bowel disease, obesity, and other bacteria-related diseases. ${ }^{2,136,137}$ Furthermore, it is regarded as a breakthrough in medical research. The safety profile of FMT has also been demonstrated in a number of studies. In an academic medical center, Navalkele et al performed a retention enema for fecal microbiota transplantation in 47 patients with recurrent Clostridioides difficile infections, including $17 \mathrm{immu-}$ nocompromised patients, which proved safe and 


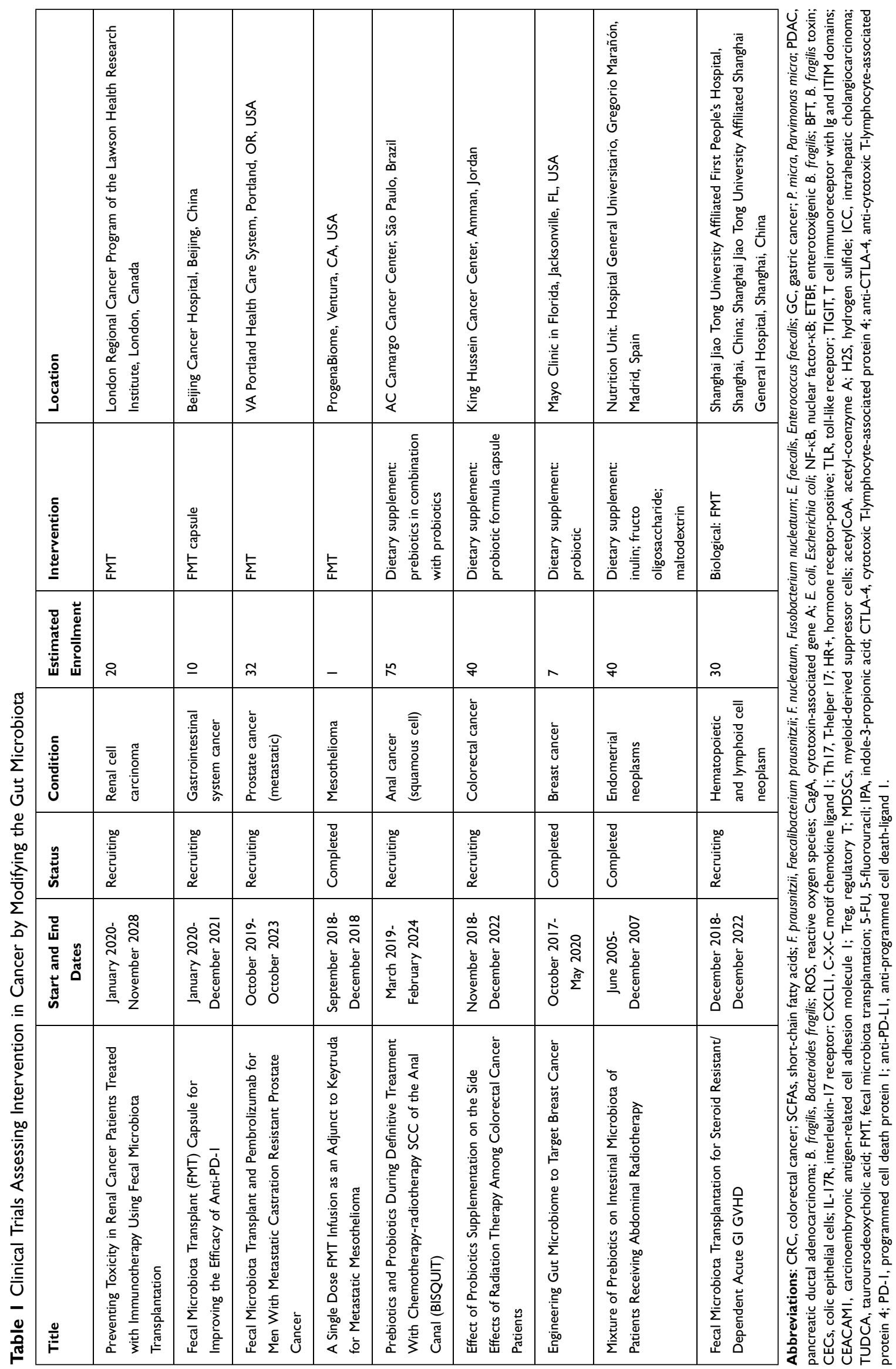


effective. ${ }^{138}$ In 2017, a male patient with Philadelphiapositive acute lymphoblastic leukemia had a serious infection ( $\beta$-lactamase-producing E. coli, Clostridium difficile, and carbapenemase-producing Enterobacteria) that occurred before preparation for hematopoietic stem cell transplantation; the symptoms of the infection improved after undergoing FMT. ${ }^{139}$

Currently, research is focused on a more targeted approach, namely the precise adjustment of the gut microbiota. For instance, this approach involves the development of a drug that specifically targets Fusobacterium, the production of a vaccine, or the use of phage infection to precisely destroy this harmful microorganism. ${ }^{118}$ Some researchers are concerned that toxic microbes may pass through the screening process. Therefore, they are focusing on well-characterized and laboratory-grown microbial formulations that use properly designed combinations of bacteria. In addition to their specific safety advantages, these microbial formulations can also be modified and extended by evaluating performance and other indicators. ${ }^{140}$

\section{Prospects and Challenges}

Over the years, numerous studies have shown that the gut microbiota is inextricably linked to cancer. Overall, researchers have found significant changes in the gut microbiota of patients with various cancers, in which specific bacteria and/or microbiota characteristics may serve as biomarkers for cancer screening and prognosis prediction. The gut microbiota influences the occurrence and development of cancer through mutation, metabolism, immunity, and other pathways. Regulating gut microbes through diet, probiotics, FMT, and more can influence cancer and response to treatment. With the development of metagenomics and metabolomics deep sequencing technology, and the establishment of multidisciplinary collaborative networks, research on the gut microbiota is continuously expanding. This lays a solid foundation for investigators to better understand the composition and function of this complex ecosystem. Meanwhile, decoding the relationships among intestinal flora, metabolism, immune system, cancer progression, and response to treatment will deepen the current understanding of the mechanism of cancer development.

Despite intensive efforts, there are numerous challenges to overcome. Most experts acknowledge that the causal relationship between the human microbiome and cancer remains to be determined. Current microbiome studies lack prospective cohort designs. Hence, it is impossible to determine the role of the gut microbiota in the initiation and development of diseases. Meanwhile, different geographic populations exhibit different microbial compositions, the fecal, luminal, and mucosal microbiota vary greatly, and many intestinal bacteria are not culturable. ${ }^{141,142}$ Microbiome research studies have a high degree of heterogeneity in terms of descriptive methods, techniques used, depth of classification, and lack of information on confounders. Therefore, standardization of microbiome research methods (from sample collection to bioinformatics analysis) is urgently needed to improve the comparability of findings. Furthermore, current studies are not characterized by sufficiently high resolution to identify individual microbial species or communities that are carcinogenic and tumor suppressing. Thus, these investigations are frequently limited to recognizing associations between diseases and phyla and genera. ${ }^{143}$ In addition, although probiotics are generally considered safe, sepsis has been observed in severely immunocompromised individuals following supplementation with Bacillus subtilis, as well as in critically ill patients who received Lactobacillus rhamnosus GG. ${ }^{144}$ Therefore, the safety of usage of live bacteria for the treatment of cancer warrants further investigation. Finally, numerous experiments reported thus far were conducted on animals; hence, we should be cautious in extrapolating these results to humans.

Screening of specific gut microorganisms from different cancers as biomarkers to assess the risk and/or extent of disease in patients, as well as develop new, simple, and highly sensitive non-invasive tests has shown great potential. The prevention of cancer by fine-tuning the gut microbiota may be another adjuvant or primary therapy with great potential after surgery, radiotherapy, chemotherapy, or targeted therapy, paving the way for improved outcomes in such patients.

\section{Funding}

This research was supported by the National Natural Science Foundation of China (Grant No. 00022986) and the Natural Science Foundation of Southwest Medical University (Grant No. 2016XNYD217 and No. 2018ZRQN-032); the Science and Technology Projects of Sichuan Province (Grant No. 2018JY0403); the Sichuan Province Returnees'Science and Technology Activities 
Project (2019(76)-72); and the Scientific Research Program of Southwest Medical University (JG201977).

\section{Disclosure}

The authors report no conflicts of interest in this work.

\section{References}

1. Sung H, Ferlay J, Siegel RL, et al. Global cancer statistics 2020: GLOBOCAN estimates of incidence and mortality worldwide for 36 cancers in 185 countries. CA Cancer J Clin. 2021;71 (3):209-249. doi:10.3322/caac.21660

2. Zitvogel L, Daillere R, Roberti MP, et al. Anticancer effects of the microbiome and its products. Nat Rev Microbiol. 2017;15 (8):465-478. doi:10.1038/nrmicro.2017.44

3. Dzutsev A, Badger JH, Perez-Chanona E, et al. Microbes and Cancer. Annu Rev Immunol. 2017;35:199-228. doi:10.1146/ annurev-immunol-051116-052133

4. Yu D, Yu X, Ye A, et al. Profiling of gut microbial dysbiosis in adults with myeloid leukemia. FEBS Open Bio. 2021;11 (7):2050-2059. doi:10.1002/2211-5463.13193

5. Xie YH, Gao QY, Cai GX, et al. Fecal clostridium symbiosum for noninvasive detection of early and advanced colorectal cancer: test and validation studies. EBioMedicine. 2017;25:32-40. doi:10.1016/j.ebiom.2017.10.005

6. Roy S, Trinchieri G. Microbiota: a key orchestrator of cancer therapy. Nat Rev Cancer. 2017;17(5):271-285. doi:10.1038/nrc.2017.13

7. Garrett WS. Cancer and the microbiota. Science. 2015;348 (6230):80-86. doi:10.1126/science.aaa4972

8. Helmink BA, Khan MAW, Hermann A, et al. The microbiome, cancer, and cancer therapy. Nat Med. 2019;25(3):377-388. doi:10.1038/s41591-019-0377-7

9. Gopalakrishnan V, Helmink BA, Spencer CN, et al. The influence of the gut microbiome on cancer, immunity, and cancer immunotherapy. Cancer Cell. 2018;33(4):570-580. doi:10.1016/ j.ccell.2018.03.015

10. Rossi P, Difrancia R, Quagliariello V, et al. B-glucans from Grifola frondosa and Ganoderma lucidum in breast cancer: an example of complementary and integrative medicine. Oncotarget. 2018;9(37):24837-24856. doi:10.18632/oncotarget.24984

11. Rodriguez JM, Murphy K, Stanton C, et al. The composition of the gut microbiota throughout life, with an emphasis on early life. Microb Ecol Health Dis. 2015;26:26050.

12. Tropini C, Earle KA, Huang KC, et al. The gut microbiome: connecting spatial organization to function. Cell Host Microbe. 2017;21(4):433-442. doi:10.1016/j.chom.2017.03.010

13. Friedman ES, Bittinger K, Esipova TV, et al. Microbes vs. chemistry in the origin of the anaerobic gut lumen. Proc Natl Acad Sci US A. 2018;115(16):4170-4175. doi:10.1073/pnas. 1718635115

14. Martinez-Guryn K, Leone V, Chang EB. Regional diversity of the gastrointestinal microbiome. Cell Host Microbe. 2019;26 (3):314-324. doi:10.1016/j.chom.2019.08.011

15. Allegra A, Musolino C, Tonacci A, et al. Interactions between the MicroRNAs and Microbiota in Cancer Development: roles and Therapeutic Opportunities. Cancers. 2020;12(4):805. doi:10.3390/cancers 12040805

16. Lu K, Mahbub R, Fox JG. Xenobiotics: interaction with the Intestinal Microflora. ILAR J. 2015;56(2):218-227. doi:10.1093/ ilar/ilv018

17. Chen P, Chen X, Hao L, et al. The bioavailability of soybean polysaccharides and their metabolites on gut microbiota in the simulator of the human intestinal microbial ecosystem (SHIME). Food Chem. 2021;362:130233. doi:10.1016/j.foodchem.2021.130233
18. van der Hee B, Wells JM. Microbial regulation of host physiology by short-chain fatty acids. Trends Microbiol. 2021;29(8):700-712. doi:10.1016/j.tim.2021.02.001

19. Bozzi Cionci N, Baffoni L, Gaggia F, et al. Therapeutic microbiology: the role of bifidobacterium breve as food supplement for the prevention/treatment of paediatric diseases. Nutrients. 2018;10(11):1723. doi:10.3390/nu10111723

20. Fragkou PC, Karaviti D, Zemlin M, et al. Impact of early life nutrition on children's immune system and noncommunicable diseases through its effects on the bacterial microbiome, virome and mycobiome. Front Immunol. 2021;12:644269. doi:10.3389/ fimmu.2021.644269

21. Zhao Y, Liu Y, Li S, et al. Role of lung and gut microbiota on lung cancer pathogenesis. J Cancer Res Clin Oncol. 2021;147 (8):2177-2186. doi:10.1007/s00432-021-03644-0

22. Kim D, Zeng MY, Nunez G. The interplay between host immune cells and gut microbiota in chronic inflammatory diseases. Exp Mol Med. 2017;49(5):e339. doi:10.1038/emm.2017.24

23. Ogbonnaya ES, Clarke G, Shanahan F, et al. Adult hippocampal neurogenesis is regulated by the microbiome. Biol Psychiatry. 2015;78(4):e7-e9. doi:10.1016/j.biopsych.2014.12.023

24. Codagnone MG, Stanton C, O'Mahony SM, et al. Microbiota and Neurodevelopmental trajectories: role of maternal and early-life nutrition. Ann Nutr Metab. 2019;74(Suppl 2):16-27. doi:10.1159/ 000499144

25. Strandwitz P. Neurotransmitter modulation by the gut microbiota. Brain Res. 2018;1693(Pt $\quad$ B):128-133. doi:10.1016/j. brainres.2018.03.015

26. Louis P, Flint HJ. Formation of propionate and butyrate by the human colonic microbiota. Environ Microbiol. 2017;19(1):29-41. doi:10.1111/1462-2920.13589

27. Paul B, Barnes S, Demark-Wahnefried W, et al. Influences of diet and the gut microbiome on epigenetic modulation in cancer and other diseases. Clin Epigenetics. 2015;7:112. doi:10.1186/s13148015-0144-7

28. Sampsell K, Hao D, Reimer RA. The gut microbiota: a potential gateway to improved health outcomes in breast cancer treatment and survivorship. Int J Mol Sci. 2020;21(23):9239. doi:10.3390/ ijms21239239

29. Pugin B, Barcik W, Westermann P, et al. A wide diversity of bacteria from the human gut produces and degrades biogenic amines. Microb Ecol Health Dis. 2017;28(1):1353881.

30. Magnúsdóttir S, Ravcheev D, de Crécy-lagard V, et al. Systematic genome assessment of B-vitamin biosynthesis suggests co-operation among gut microbes. Front Genet. 2015;6:148. doi:10.3389/fgene.2015.00148

31. Schwabe RF, Jobin C. The microbiome and cancer. Nat Rev Cancer. 2013;13(11):800-812. doi:10.1038/nrc3610

32. Levy M, Kolodziejczyk AA, Thaiss CA, et al. Dysbiosis and the immune system. Nat Rev Immunol. 2017;17(4):219-232. doi:10.1038/nri.2017.7

33. Parida S, Sharma D. The microbiome and cancer: creating friendly neighborhoods and removing the foes within. Cancer Res. 2021;81(4):790-800. doi:10.1158/0008-5472.CAN-20-2629

34. Vivarelli S, Salemi R, Candido S, et al. Gut microbiota and cancer: from pathogenesis to therapy. Cancers. 2019;11(1):38. doi:10.3390/cancers 11010038

35. Khan FH, Bhat BA, Sheikh BA, et al. Microbiome dysbiosis and epigenetic modulations in lung cancer: from pathogenesis to therapy. Semin Cancer Biol. 2021. doi:10.1016/j.semcancer.2021.07.005

36. Villeger R, Lopes A, Veziant J, et al. Microbial markers in colorectal cancer detection and/or prognosis. World $J$ Gastroenterol. 2018;24(22):2327-2347. doi:10.3748/wjg.v24.i22.2327

37. Amieva M, Peek RM Jr. Pathobiology of helicobacter pylori-induced gastric cancer. Gastroenterology. 2016;150 (1):64-78. doi:10.1053/j.gastro.2015.09.004 
38. Yu T, Guo F, Yu Y, et al. Fusobacterium nucleatum Promotes Chemoresistance to Colorectal Cancer by Modulating Autophagy. Cell. 2017;170(3):548-563 e516. doi:10.1016/j.cell.2017.07.008

39. Feng J, Zhao F, Sun J, et al. Alterations in the gut microbiota and metabolite profiles of thyroid carcinoma patients. Int $J$ Cancer. 2019;144(11):2728-2745. doi:10.1002/ijc.32007

40. Goedert JJ, Jones G, Hua X, et al. Investigation of the association between the fecal microbiota and breast cancer in postmenopausal women: a population-based case-control pilot study. J Natl Cancer Inst. 2015;107:8. doi:10.1093/jnci/djv147

41. Vernocchi P, Gili T, Conte F, et al. Network analysis of gut microbiome and metabolome to discover microbiota-linked biomarkers in patients affected by non-small cell lung cancer. Int J Mol Sci. 2020;21(22):8730. doi:10.3390/ijms21228730

42. Zheng Y, Fang Z, Xue Y, et al. Specific gut microbiome signature predicts the early-stage lung cancer. Gut Microbes. 2020;11 (4):1030-1042. doi:10.1080/19490976.2020.1737487

43. Coker OO, Dai Z, Nie Y, et al. Mucosal microbiome dysbiosis in gastric carcinogenesis. Gut. 2018;67(6):1024-1032. doi:10.1136/ gutjnl-2017-314281

44. Hsieh YY, Tung SY, Pan HY, et al. Increased Abundance of Clostridium and Fusobacterium in Gastric Microbiota of Patients with Gastric Cancer in Taiwan. Sci Rep. 2018;8(1):158. doi:10.1038/s41598-017-18596-0

45. Ferreira RM, Pereira-Marques J, Pinto-Ribeiro I, et al. Gastric microbial community profiling reveals a dysbiotic cancer-associated microbiota. Gut. 2018;67(2):226-236. doi:10.1136/gutjnl-2017-314205

46. Zhang Y, Shen J, Shi X, et al. Gut microbiome analysis as a predictive marker for the gastric cancer patients. Appl Microbiol Biotechnol. 2021;105(2):803-814. doi:10.1007/ s00253-020-11043-7

47. Pushalkar S, Hundeyin M, Daley D, et al. The pancreatic cancer microbiome promotes oncogenesis by induction of innate and adaptive immune suppression. Cancer Discov. 2018;8 (4):403-416. doi:10.1158/2159-8290.CD-17-1134

48. Yang J, Li D, Yang Z, et al. Establishing high-accuracy biomarkers for colorectal cancer by comparing fecal microbiomes in patients with healthy families. Gut Microbes. 2020;11 (4):918-929. doi:10.1080/19490976.2020.1712986

49. Thomas AM, Manghi P, Asnicar F, et al. Metagenomic analysis of colorectal cancer datasets identifies cross-cohort microbial diagnostic signatures and a link with choline degradation. Nat Med. 2019;25(4):667-678. doi:10.1038/s41591-019-0405-7

50. Kostic AD, Gevers D, Pedamallu CS, et al. Genomic analysis identifies association of Fusobacterium with colorectal carcinoma. Genome Res. 2012;22(2):292-298. doi:10.1101/gr.126573.111

51. Kostic AD, Chun E, Robertson L, et al. Fusobacterium nucleatum potentiates intestinal tumorigenesis and modulates the tumor-immune microenvironment. Cell Host Microbe. 2013;14 (2):207-215. doi:10.1016/j.chom.2013.07.007

52. Gagniere J, Raisch J, Veziant J, et al. Gut microbiota imbalance and colorectal cancer. World J Gastroenterol. 2016;22 (2):501-518. doi:10.3748/wjg.v22.i2.501

53. Wu N, Yang X, Zhang R, et al. Dysbiosis signature of fecal microbiota in colorectal cancer patients. Microb Ecol. 2013;66 (2):462-470. doi:10.1007/s00248-013-0245-9

54. Panebianco C, Andriulli A, Pazienza V. Pharmacomicrobiomics: exploiting the drug-microbiota interactions in anticancer therapies. Microbiome. 2018;6(1):92. doi:10.1186/s40168-0180483-7

55. Whisner CM, Athena Aktipis C. The Role of the Microbiome in Cancer Initiation and Progression: how Microbes and Cancer Cells Utilize Excess Energy and Promote One Another's Growth. Curr Nutr Rep. 2019;8(1):42-51. doi:10.1007/s13668019-0257-2
56. Ogrendik M. Periodontal pathogens in the etiology of pancreatic cancer. Gastrointest Tumors. 2017;3(3-4):125-127. doi:10.1159/ 000452708

57. Wilson MR, Jiang Y, Villalta PW, et al. The human gut bacterial genotoxin colibactin alkylates DNA. Science. 2019;363:6428. doi:10.1126/science.aar7785

58. Scott AJ, Alexander JL, Merrifield CA, et al. International Cancer Microbiome Consortium consensus statement on the role of the human microbiome in carcinogenesis. Gut. 2019;68 (9):1624-1632. doi:10.1136/gutjnl-2019-318556

59. Nguyen LH, Ma W, Wang DD, et al. Association between sulfur-metabolizing bacterial communities in stool and risk of distal colorectal cancer in men. Gastroenterology. 2020;158 (5):1313-1325. doi:10.1053/j.gastro.2019.12.029

60. Andriamihaja M, Lan A, Beaumont M, et al. The deleterious metabolic and genotoxic effects of the bacterial metabolite p-cresol on colonic epithelial cells. Free Radic Biol Med. 2015;85:219-227. doi:10.1016/j.freeradbiomed.2015.04.004

61. Tahara T, Hirata I, Nakano N, et al. Potential link between Fusobacterium enrichment and DNA methylation accumulation in the inflammatory colonic mucosa in ulcerative colitis. Oncotarget. 2017;8(37):61917-61926. doi:10.18632/oncotarget.18716

62. Afify SM, Seno M. Conversion of stem cells to cancer stem cells: undercurrent of cancer initiation. Cancers. 2019;11(3). doi:10.3390/cancers 11030345

63. Yousefi B, Mohammadlou M, Abdollahi M, et al. Epigenetic changes in gastric cancer induction by Helicobacter pylori. $J$ Cell Physiol. 2019;234(12):21770-21784. doi:10.1002/ jcp. 28925

64. Caballero S, Pamer EG. Microbiota-mediated inflammation and antimicrobial defense in the intestine. Annu Rev Immunol. 2015;33:227-256. doi:10.1146/annurev-immunol $-032713-120238$

65. Wei MY, Shi S, Liang C, et al. The microbiota and microbiome in pancreatic cancer: more influential than expected. Mol Cancer. 2019;18(1):97. doi:10.1186/s12943-019-1008-0

66. Chung L, Thiele Orberg E, Geis AL, et al. Bacteroides fragilis Toxin Coordinates a Pro-carcinogenic Inflammatory Cascade via Targeting of Colonic Epithelial Cells. Cell Host Microbe. 2018;23 (2):203-214 e205. doi:10.1016/j.chom.2018.01.007

67. Buchta Rosean C, Bostic RR, Ferey JCM, et al. Preexisting commensal dysbiosis is a host-intrinsic regulator of tissue inflammation and tumor cell dissemination in hormone receptor-positive breast cancer. Cancer Res. 2019;79(14):3662-3675. doi:10.1158/ 0008-5472.CAN-18-3464

68. Rutkowski MR, Svoronos N, Perales-Puchalt A, et al. The tumor macroenvironment: cancer-promoting networks beyond tumor beds. Adv Cancer Res. 2015;128:235-262.

69. Wong DV, Lima-Junior RC, Carvalho CB, et al. The Adaptor protein myd88 is a key signaling molecule in the pathogenesis of irinotecan-induced intestinal mucositis. PLoS One. 2015;10(10): e0139985. doi:10.1371/journal.pone.0139985

70. Albulescu R, Codrici E, Popescu ID, et al. Cytokine patterns in brain tumour progression. Mediators Inflamm. 2013;2013:979748. doi:10.1155/2013/979748

71. Qiu Q, Lin Y, Ma Y, et al. Exploring the emerging role of the gut microbiota and tumor microenvironment in cancer immunotherapy. Front Immunol. 2020;11:612202. doi:10.3389/ fimmu.2020.612202

72. Ducimetiere L, Vermeer M, Tugues S. The Interplay Between Innate Lymphoid Cells and the Tumor Microenvironment. Front Immunol. 2019;10:2895. doi:10.3389/fimmu.2019.02895

73. Gur C, Ibrahim Y, Isaacson B, et al. Binding of the Fap2 protein of Fusobacterium nucleatum to human inhibitory receptor TIGIT protects tumors from immune cell attack. Immunity. 2015;42 (2):344-355. doi:10.1016/j.immuni.2015.01.010 
74. Gur C, Maalouf N, Shhadeh A, et al. Fusobacterium nucleatum supresses anti-tumor immunity by activating CEACAM1. Oncoimmunology. 2019;8(6):e1581531. doi:10.1080/ 2162402X.2019.1581531

75. Rubinstein MR, Baik JE, Lagana SM, et al. Fusobacterium nucleatum promotes colorectal cancer by inducing Wnt/beta-catenin modulator Annexin A1. EMBO Rep. 2019;20(4). doi:10.15252/ embr.201847638

76. Zhang Q, Ma C, Duan Y, et al. Gut Microbiome Directs Hepatocytes to Recruit MDSCs and Promote Cholangiocarcinoma. Cancer Discov. 2021;11(5):1248-1267. doi:10.1158/2159-8290.CD-20-0304

77. Pandey S, Singh S, Anang V, et al. Pattern recognition receptors in cancer progression and metastasis. Cancer Growth Metastasis. 2015;8:25-34. doi:10.4137/CGM.S24314

78. Seifert L, Werba G, Tiwari S, et al. The necrosome promotes pancreatic oncogenesis via CXCL1 and Mincle-induced immune suppression. Nature. 2016;532(7598):245-249. doi:10.1038/ nature 17403

79. Zambirinis CP, Levie E, Nguy S, et al. TLR9 ligation in pancreatic stellate cells promotes tumorigenesis. J Exp Med. 2015;212 (12):2077-2094. doi:10.1084/jem.20142162

80. Sun J, Chang EB. Exploring gut microbes in human health and disease: pushing the envelope. Genes Dis. 2014;1(2):132-139. doi:10.1016/j.gendis.2014.08.001

81. Deng L, Wang R, Li H, et al. miRNA-Gene Regulatory Network in Gnotobiotic Mice Stimulated by Dysbiotic Gut Microbiota Transplanted From a Genetically Obese Child. Front Microbiol. 2019;10:1517. doi:10.3389/fmicb.2019.01517

82. Hoban AE, Stilling RM. Microbial regulation of microRNA expression in the amygdala and prefrontal cortex. Microbiome. 2017;5(1):102. doi:10.1186/s40168-017-0321-3

83. Louis P, Hold GL, Flint HJ. The gut microbiota, bacterial metabolites and colorectal cancer. Nat Rev Microbiol. 2014;12 (10):661-672. doi:10.1038/nrmicro3344

84. Ha CW, Lam YY, Holmes AJ. Mechanistic links between gut microbial community dynamics, microbial functions and metabolic health. World J Gastroenterol. 2014;20(44):16498-16517. doi:10.3748/wjg.v20.i44.16498

85. Wang T, Cai G, Qiu Y, et al. Structural segregation of gut microbiota between colorectal cancer patients and healthy volunteers. ISME J. 2012;6(2):320-329. doi:10.1038/ismej.2011.109

86. Farhana L, Nangia-Makker P, Arbit E, et al. Bile acid: a potential inducer of colon cancer stem cells. Stem Cell Res Ther. 2016;7 (1):181. doi:10.1186/s13287-016-0439-4

87. Smith PM, Howitt MR, Panikov N, et al. The microbial metabolites, short-chain fatty acids, regulate colonic Treg cell homeostasis. Science. 2013;341(6145):569-573. doi:10.1126/ science. 1241165

88. Lee JV, Berry CT, Kim K, et al. Acetyl-CoA promotes glioblastoma cell adhesion and migration through $\mathrm{Ca}(2+)$-NFAT signaling. Genes Dev. 2018;32(7-8):497-511. doi:10.1101/ gad.311027.117

89. Lu M, Zhu WW, Wang X, et al. ACOT12-Dependent Alteration of Acetyl-CoA Drives Hepatocellular Carcinoma Metastasis by Epigenetic Induction of Epithelial-Mesenchymal Transition. Cell Metab. 2019;29(4):886-900 e885. doi:10.1016/ j.cmet.2018.12.019

90. Schug ZT, Peck B, Jones DT, et al. Acetyl-CoA synthetase 2 promotes acetate utilization and maintains cancer cell growth under metabolic stress. Cancer Cell. 2015;27(1):57-71. doi:10.1016/j.ccell.2014.12.002

91. Wallace JL, Blackler RW, Chan MV, et al. Anti-inflammatory and cytoprotective actions of hydrogen sulfide: translation to therapeutics. Antioxid Redox Signal. 2015;22(5):398-410. doi:10.1089/ars.2014.5901
92. Saint-Georges-Chaumet Y, Edeas M. Microbiota-mitochondria inter-talk: consequence for microbiota-host interaction. Pathog Dis. 2016;74(1):ftv096. doi:10.1093/femspd/ftv096

93. Ijssennagger N, van der Meer R, van Mil SWC. Sulfide as a Mucus Barrier-Breaker in Inflammatory Bowel Disease? Trends Mol Med. 2016;22(3):190-199. doi:10.1016/j. molmed.2016.01.002

94. Wolf AMD, Fontham ETH, Church TR, et al. Colorectal cancer screening for average-risk adults: 2018 guideline update from the American Cancer Society. CA Cancer J Clin. 2018;68 (4):250-281. doi:10.3322/caac.21457

95. Wu Y, Jiao N, Zhu R, et al. Identification of microbial markers across populations in early detection of colorectal cancer. Nat Commun. 2021;12(1):3063. doi:10.1038/s41467-021-23265-y

96. Luu TH, Michel C, Bard JM, et al. Intestinal Proportion of Blautia sp. is Associated with Clinical Stage and Histoprognostic Grade in Patients with Early-Stage Breast Cancer. Nutr Cancer. 2017;69(2):267-275. doi:10.1080/ 01635581.2017.1263750

97. Jia X, Lu S, Zeng Z, et al. Characterization of Gut Microbiota, Bile Acid Metabolism, and Cytokines in Intrahepatic Cholangiocarcinoma. Hepatology. 2020;71(3):893-906. doi:10.1002/hep.30852

98. Haghi F, Goli E, Mirzaei B, et al. The association between fecal enterotoxigenic B. fragilis with colorectal cancer. BMC Cancer. 2019;19(1):879. doi:10.1186/s12885-019-6115-1

99. Lowenmark T, Lofgren-Burstrom A, Zingmark $\mathrm{C}$, et al. Parvimonas micra as a putative non-invasive faecal biomarker for colorectal cancer. Sci Rep. 2020;10(1):15250. doi:10.1038/ s41598-020-72132-1

100. Khan DA. Proactive management of penicillin and other antibiotic allergies. Allergy Asthma Proc. 2020;41(2):82-89. doi:10.2500/aap.2020.41.190024

101. Iqbal U, Anwar H, Karim MA. Safety and efficacy of encapsulated fecal microbiota transplantation for recurrent Clostridium difficile infection: a systematic review. Eur J Gastroenterol Hepatol. 2018;30(7):730-734. doi:10.1097/MEG.000000 0000001147

102. Babjuk M, Burger M, Comperat EM, et al. European Association of Urology Guidelines on Non-muscle-invasive Bladder Cancer (TaT1 and Carcinoma In Situ) - 2019 Update. Eur Urol. 2019;76 (5):639-657. doi:10.1016/j.eururo.2019.08.016

103. Alexander JL, Wilson ID, Teare J, et al. Gut microbiota modulation of chemotherapy efficacy and toxicity. Nat Rev Gastroenterol Hepatol. 2017;14(6):356-365. doi:10.1038/nrgastro.2017.20

104. Daillere R, Vetizou M, Waldschmitt N, et al. Enterococcus hirae and barnesiella intestinihominis facilitate cyclophosphamide-induced therapeutic immunomodulatory effects. Immunity. 2016;45 (4):931-943. doi:10.1016/j.immuni.2016.09.009

105. Viaud S, Saccheri F, Mignot G, et al. The intestinal microbiota modulates the anticancer immune effects of cyclophosphamide. Science. 2013;342(6161):971-976. doi:10.1126/science.1240537

106. Iida N, Dzutsev A, Stewart CA, et al. Commensal bacteria control cancer response to therapy by modulating the tumor microenvironment. Science. 2013;342(6161):967-970. doi:10.1126/science. 1240527

107. Shen S, Lim G, You Z, et al. Gut microbiota is critical for the induction of chemotherapy-induced pain. Nat Neurosci. 2017;20 (9):1213-1216. doi:10.1038/nn.4606

108. Ding C, Tang W, Fan X, et al. Intestinal microbiota: a novel perspective in colorectal cancer biotherapeutics. Onco Targets Ther. 2018;11:4797-4810. doi:10.2147/OTT.S170626

109. Yan A, Culp E, Perry J, et al. Transformation of the anticancer drug doxorubicin in the human gut microbiome. ACS Infect Dis. 2018;4(1):68-76. doi:10.1021/acsinfecdis.7b00166 
110. Geller LT, Barzily-Rokni M, Danino T, et al. Potential role of intratumor bacteria in mediating tumor resistance to the chemotherapeutic drug gemcitabine. Science. 2017;357 (6356):1156-1160. doi:10.1126/science.aah5043

111. Uribe-Herranz M, Rafail S, Beghi S, et al. Gut microbiota modulate dendritic cell antigen presentation and radiotherapy-induced antitumor immune response. J Clin Invest. 2020;130(1):466-479. doi:10.1172/JCI124332

112. Alexeev EE, Lanis JM, Kao DJ, et al. Microbiota-Derived Indole Metabolites Promote Human and Murine Intestinal Homeostasis through Regulation of Interleukin-10 Receptor. Am J Pathol. 2018;188(5):1183-1194. doi:10.1016/j.ajpath.2018.01.011

113. Xiao HW, Cui M, Li Y, et al. Gut microbiota-derived indole 3 -propionic acid protects against radiation toxicity via retaining acyl-CoA-binding protein. Microbiome. 2020;8(1):69. doi:10.1186/s40168-020-00845-6

114. Reis Ferreira M, Andreyev HJN, Mohammed K, et al. Microbiota- and Radiotherapy-Induced Gastrointestinal Side-Effects (Mars) Study: a Large Pilot Study of the Microbiome in Acute and Late-Radiation Enteropathy. Clin Cancer Res. 2019;25(21):6487-6500. doi:10.1158/1078-0432. CCR-19-0960

115. Kennedy LB, Salama AKS. A review of cancer immunotherapy toxicity. CA Cancer J Clin. 2020;70(2):86-104. doi:10.3322/ caac. 21596

116. Vétizou M, Pitt JM, Daillère R, et al. Anticancer immunotherapy by CTLA-4 blockade relies on the gut microbiota. Science. 2015;350(6264):1079-1084. doi:10.1126/science.aad1329

117. Sivan A, Corrales L, Hubert N, et al. Commensal Bifidobacterium promotes antitumor immunity and facilitates anti-PD-L1 efficacy. Science. 2015;350(6264):1084-1089. doi:10.1126/science. aac4255

118. Gopalakrishnan V, Spencer CN, Nezi L, et al. Gut microbiome modulates response to anti-PD-1 immunotherapy in melanoma patients. Science. 2018;359(6371):97-103. doi:10.1126/science. aan4236

119. Derosa L, Hellmann MD, Spaziano M, et al. Negative association of antibiotics on clinical activity of immune checkpoint inhibitors in patients with advanced renal cell and non-small-cell lung cancer. Ann Oncol. 2018;29(6):1437-1444. doi:10.1093/annonc/ mdy 103

120. Routy B, Le Chatelier E, Derosa L, et al. Gut microbiome influences efficacy of PD-1-based immunotherapy against epithelial tumors. Science. 2018;359(6371):91-97. doi:10.1126/science. aan3706

121. Frankel AE, Coughlin LA, Kim J, et al. Metagenomic Shotgun Sequencing and Unbiased Metabolomic Profiling Identify Specific Human Gut Microbiota and Metabolites Associated with Immune Checkpoint Therapy Efficacy in Melanoma Patients. Neoplasia. 2017;19(10):848-855. doi:10.1016/j. neo.2017.08.004

122. Temraz S, Nassar F, Nasr R, et al. Gut Microbiome: a Promising Biomarker for Immunotherapy in Colorectal Cancer. Int $J \mathrm{Mol}$ Sci. 2019;20:17. doi:10.3390/ijms20174155

123. So D, Whelan K, Rossi M, et al. Dietary fiber intervention on gut microbiota composition in healthy adults: a systematic review and meta-analysis. Am J Clin Nutr. 2018;107(6):965-983. doi:10.1093/ajen/nqy041

124. Mendez Utz VE, Perez Visnuk D, Perdigon G, et al. Milk fermented by Lactobacillus casei CRL431 administered as an immune adjuvant in models of breast cancer and metastasis under chemotherapy. Appl Microbiol Biotechnol. 2021;105 (1):327-340. doi:10.1007/s00253-020-11007-x

125. Bhatt AP, Redinbo MR, Bultman SJ. The role of the microbiome in cancer development and therapy. CA Cancer J Clin. 2017;67 (4):326-344. doi:10.3322/caac.21398
126. Taherian M, Mahin Samadi P, Rastegar H, et al. An Overview on Probiotics as an Alternative Strategy for Prevention and Treatment of Human Diseases. Iran J Pharm Res. 2019;18 (Suppl1):31-50.

127. Al-Sadi R, Nighot P, Nighot $M$, et al. Lactobacillus acidophilus Induces a Strain-specific and Toll-Like Receptor 2-Dependent Enhancement of Intestinal Epithelial Tight Junction Barrier and Protection Against Intestinal Inflammation. Am J Pathol. 2021;191(5):872-884. doi:10.1016/j.ajpath.2021.02.003

128. Al-Sadi R, Dharmaprakash V, Nighot P, et al. Bifidobacterium bifidum Enhances the Intestinal Epithelial Tight Junction Barrier and Protects against Intestinal Inflammation by Targeting the Toll-like Receptor-2 Pathway in an NF-kappaB-Independent Manner. Int J Mol Sci. 2021;22:15. doi:10.3390/ijms22158070

129. Asadollahi P, Ghanavati R, Rohani M, et al. Anti-cancer effects of Bifidobacterium species in colon cancer cells and a mouse model of carcinogenesis. PLoS One. 2020;15(5):e0232930. doi:10.1371/ journal.pone.0232930

130. Chou YC, Ho PY, Chen WJ, et al. Lactobacillus fermentum V3 ameliorates colitis-associated tumorigenesis by modulating the gut microbiome. Am J Cancer Res. 2020;10(4):1170-1181.

131. Chung Y, Ryu Y, An BC, et al. A synthetic probiotic engineered for colorectal cancer therapy modulates gut microbiota. Microbiome. 2021;9(1):122. doi:10.1186/s40168-021-01071-4

132. Ghidini M, Nicoletti M, Ratti M, et al. Lactobacillus Kefiri LKF01 (Kefibios $((\mathrm{R}))$ ) for Prevention of Diarrhoea in Cancer Patients Treated with Chemotherapy: a Prospective Study. Nutrients. 2021;13(2):385. doi:10.3390/nu13020385

133. Gibson GR, Hutkins R, Sanders ME, et al. Expert consensus document: the International Scientific Association for Probiotics and Prebiotics (ISAPP) consensus statement on the definition and scope of prebiotics. Nat Rev Gastroenterol Hepatol. 2017;14 (8):491-502. doi:10.1038/nrgastro.2017.75

134. Li Y, Elmen L, Segota I, et al. Prebiotic-Induced Anti-tumor Immunity Attenuates Tumor Growth. Cell Rep. 2020;30 (6):1753-1766 e1756. doi:10.1016/j.celrep.2020.01.035

135. Bressa C, Bailen-Andrino M, Perez-Santiago J, et al. Differences in gut microbiota profile between women with active lifestyle and sedentary women. PLoS One. 2017;12(2):e0171352. doi:10.1371/ journal.pone.0171352

136. McKenney PT, Pamer EG. From Hype to Hope: the Gut Microbiota in Enteric Infectious Disease. Cell. 2015;163 (6):1326-1332. doi:10.1016/j.cell.2015.11.032

137. Moayyedi P, Surette MG, Kim PT, et al. Fecal microbiota transplantation induces remission in patients with active ulcerative colitis in a randomized controlled trial. Gastroenterology. 2015;149(1):102-109 e106. doi:10.1053/j.gastro.2015.04.001

138. Navalkele BD, Polistico J, Sandhu A, et al. Clinical outcomes after faecal microbiota transplant by retention enema in both immunocompetent and immunocompromised patients with recurrent Clostridioides difficile infections at an academic medical centre. J Hosp Infect. 2020;106(4):643-648. doi:10.1016/j. jhin.2020.09.027

139. Innes AJ, Mullish BH, Fernando F, et al. Faecal microbiota transplant: a novel biological approach to extensively drug-resistant organism-related non-relapse mortality. Bone Marrow Transplant. 2017;52(10):1452-1454. doi:10.1038/ bmt.2017.151

140. Dolgin E. Fighting cancer with microbes. Nature. 2020;577 (7792):S16-S18. doi:10.1038/d41586-020-00199-X

141. Cavadas B, Camacho R, Ferreira JC, et al. Gastric Microbiome Diversities in Gastric Cancer Patients from Europe and Asia Mimic the Human Population Structure and Are Partly Driven by Microbiome Quantitative Trait Loci. Microorganisms. 2020;8:8. doi:10.3390/microorganisms 8081196 
142. Nayfach S, Shi ZJ, Seshadri R, et al. New insights from uncultivated genomes of the global human gut microbiome. Nature. 2019;568(7753):505-510. doi:10.1038/s41586-019-1058-x

143. Hugon P, Lagier JC, Colson P, et al. Repertoire of human gut microbes. Microb Pathog. 2017;106:103-112. doi:10.1016/j. micpath.2016.06.020
144. Sotoudegan F, Daniali M, Hassani S, et al. Reappraisal of probiotics' safety in human. Food Chem Toxicol. 2019;129:22-29. doi:10.1016/j.fct.2019.04.032

\section{Publish your work in this journal}

Cancer Management and Research is an international, peer-reviewed open access journal focusing on cancer research and the optimal use of preventative and integrated treatment interventions to achieve improved outcomes, enhanced survival and quality of life for the cancer patient.
The manuscript management system is completely online and includes a very quick and fair peer-review system, which is all easy to use. Visit http://www.dovepress.com/testimonials.php to read real quotes from published authors. 